\title{
Robots and COVID-19: Re-imagining Human-Robot Collaborative Work in Terms of Reducing Risks to Essential Workers
}

\author{
Connor Esterwood \\ School of Information \\ University of Michigan \\ 105 S. State Street \\ Ann Arbor, MI 48105 \\ $865-0257-9203$ \\ cte@umich.edu
}

\author{
Lionel P. Robert Jr. \\ School of Information \\ Robotics Institute \\ University of Michigan \\ 105 S. State Street \\ Ann Arbor, MI 48105 \\ 1probert@umich.edu
}

\begin{abstract}
The COVID-19 pandemic has led to the widespread adoption of physical distancing to prevent the disease's spread. Physical distancing, however, is not always feasible for essential workers. Robots are one proposed solution to help ensure that essential work is performed while reducing the risk of COVID-19 exposure among essential workers and their families. The COVID-19 pandemic has, however, highlighted the ability and inability of robots to directly replace human labor. At present, much of the discussion has focused on the need for technical developments in robotics. This perspective is short-sighted because it and fails to leverage the collaborative nature of work between humans and robots. In response, this article acts as a call to shift the conversation away from technical developments and toward a focus on human and robot work redesign.
\end{abstract}

Keywords: COVID-19, Robots, Labor, Collaboration, Human-Robot Interaction

Esterwood, C. \& Robert, L. P. (2021). Robots and COVID-19: Re-imagining HumanRobot Collaborative Work in Terms of Reducing Risks to Essential Workers, Robonomics: The Journal of the Automated Economy, 1(1), forthcoming. 


\section{Introduction}

According to the World Health Organization, more than 79.2 million people worldwide were infected with COVID-19, the disease caused by the coronavirus SARS-Cov-2, by late December (World Health Organization, 2020). This has led to more than 100 nations engaging in physical distancing as the primary means to combat the spread of COVID-19. Theoretically, physical distancing requires that individuals stay at least 6 feet apart to reduce the possibility of virus transmission. However, physical distancing is not always possible for essential workers. Essential workers perform tasks that are deemed vital to our society such as delivering goods, stocking and selling groceries, and providing health care services. These tasks often require close interactions with others, which limits the opportunity for essential workers to engage in physical distancing. Therefore, essential workers are often asked to place themselves and their families at risk by potentially exposing themselves to the virus.

Robots are one proposed solution to help ensure that essential work is performed while reducing the risk of essential workers and their families being exposed to COVID-19 (Seyitoglu \& Ivanov, 2020; Shen et al., 2020). Robots can be defined as artificial intelligence agents with physical embodiment (You \& Robert, 2018a). However, the COVID-19 pandemic has highlighted the ability and inability of robots to directly replace human labor. Unfortunately, much of the discussion has focused on the need for technical development in robotics. For example, the challenges highlighted in a recent review of the use of robots during the COVID19 pandemic revolve primarily around furthering a given robot's technical capabilities such as battery life, flight time, localization and perception (Shen et al., 2020). This discussion is shortsighted and fails to leverage the collaborative nature of work between humans and robots. We would like to start another discussion that acknowledges that the robot is one part of a human and robot collaborative system. This approach allows us to focus on redesigning work to create synergistic teamwork. Redesigning or re-imagining work allows us to leverage the existing robotic capabilities to reduce the risk of essential workers' exposure to COVID-19 while accomplishing the much-needed essential work.

In this article, we hope to shift this discussion in order to help essential workers deal with the ongoing COVID-19 pandemic. We recommend that governmental and other funding agencies recognize and incentivize more research focusing on human and robot work redesign. To buttress our argument for increased attention and funding to human and robot work redesign, we: (1) reflect on what has gone well with the use of robots to respond to COVID-19, (2) reflect on what has not gone well and (3) discuss the need to redesign work to better accommodate the existing robot capabilities rather than waiting for more technological development.

\section{What Has Gone Well}

According to the news media, three types of successful employment of robots have occurred during the pandemic. The first, telepresence robots have been effective at minimizing the interaction between humans while enabling embodied interactions that would have not otherwise been possible (Ackerman, 2020; Adams et al., 2020; Demaitre, 2020; Slotkin, 2020; Yang et al., 2020; Zeldovich, 2020). Telepresence robots have typically been used in health care environments, for example by allowing doctors and family members to visit people 
infected with COVID-19. Although telepresence robots are not new, the COVID-19 pandemic has spurred more enthusiasm for their wider adoption. The second has been robots that perform very narrow standalone tasks that require little or no human collaboration (Adams et al., 2020; Blake, 2020; Corkery \& Gelles, 2020; Demaitre, 2020; Field \& Murphy, 2020; Hasija \& Gumaledar, 2020; Kahn, 2020; Purtill, 2020; PYMNTS, 2019; Redman, 2019; Shin, 2020; Terra Drone, 2020; Turmelle, 2020; Vincent, 2020; Yang et al., 2020; Zeldovich, 2020). Examples of these robots include warehouse robots, scanning/counting robots, and porter robots. These robots operate in static and stable environments performing the same task over and over. The stable environment and repetitive nature of narrowly defined tasks align well with the existing capabilities of robots. Finally, delivery service robots have been successful in providing goods to customers (Biron, 2020; Marr, 2020; Tangermann, 2020). Although not widespread, their use is becoming popular and recent legislation has opened more opportunities for the use of delivery service robots (Boyle, 2020; Federal Aviation Administration, 2020).

\section{What Has Not Gone Well}

Although there have been successful deployments of robots during this pandemic, there is a reason we do not see robots taking over for essential workers on a large scale: essential workers are largely generalists performing many different types of tasks. This requires them to switch among tasks and roles fluidly throughout an average workday. A grocery store worker, for example, is at different points in time a salesperson, a stocker, a cleaner and a cashier. Robots are simply not very good at this kind of flexibility and often fail to adapt to changes or perform adequately when tasks are presented in variable and unpredictable orders (Handling, 2020; Hasija \& Gumaledar, 2020; Knight, n.d.; Simon, n.d.; Walsh, n.d.). As pointed out by Knight (n.d.), "[Robots] typically lack the ability to sense, respond, and adapt to the real world, so humans are still crucial even in the most automated facilities." Humans adapt, learn, and change on the fly and are capable of ad hoc tasks and decision-making. Robots struggle with this kind of work and find it difficult to adapt to the challenges presented to an essential worker. Therefore, the inability of robots corresponds nicely with the abilities of humans.

\section{Human and Robot Collaborative Work Redesign}

Although many lament the technical inability of robots to fully replace human workers, we believe that the redesign or re-imagining of human-robot work is a much more fruitful approach to capitalizing on the human's and robot's relative strength simultaneously. To do this, work needs to be viewed as a collaborative product of both humans and robots (Eglash et al., 2020; Robert et al., 2020; You \& Robert, 2018a). Human and robot collaborative work redesign is the process of disassembling and reassembling tasks and responsibilities between robots and humans to better align with their capabilities (Esterwood \& Robert, 2020). Human and robot collaborative work redesign requires asking and answering questions like: What tasks are better suited for robots and which tasks are better suited for humans? Can tasks be redesigned to make them more suitable for robots? What tasks can robots do with human assistance and what tasks can humans do with robot assistance? This leads to rethinking assumptions about current work practices and identifying the old ideas do that work. The main objective of human and robot collaborative work redesign is to ensure the human/robot is 
assigned to perform the right task and get the maximum performance while ensuring high levels of human satisfaction (You et al., 2018; You \& Robert, 2018b).

In line with this is the idea that robots should not replace human workers but instead help or augment them by complementing their capabilities. Humans and robots have different strengths and weaknesses. To put it simply, there are tasks that humans do well that robots cannot and should not do and vice versa. Human-robot collaborative work can capitalize on the strengths of humans and robots while mitigating human workers' exposure to COVID-19. But to leverage this, we not only need technology innovation but also work redesign innovations. This requires a co-development process between robot and work designers along with the human workers that drive them. A discussion along these lines is much more likely to lead to better exploitation of existing technology and allow us to rapidly reduce the spread of COVID-19 to our essential workers. As such, there is a need to recognize and incentivize more research that focuses on human and robot work redesign.

\section{Conclusion}

To be sure, it is not our intent to downplay the importance or need for technical development in robotics. The point is that another view of the problem might not only help us deploy robots in ways that reduce risk to essential workers sooner but also help us direct and guide such technical developments. If we could develop fully autonomous robots that perform all the tasks required of a human essential worker by tomorrow, that would be ideal, but that will not happen so quickly and COVID-19 is rapidly spreading. In the meantime, we can reduce the risk of essential workers' exposure by considering the design of human-robot teams to better leverage the existing robotic technology. To accomplish this, there is a need to direct both attention and funding toward human and robot work redesign. 


\section{References}

Ackerman, E. (2020, April). Telepresence robots are helping take pressure off hospital staff. IEEE Spectrum. https://spectrum.ieee.org/automaton/robotics/medical-robots/ telepresence-robots-are-helping-take-pressure-off-hospital-staff

Adams, J., Murphy, R. R., \& Gandudi, V. B. M. (2020, April). Robots are playing many roles in the coronavirus crisis - and offering lessons for future disasters. The Conversation. $\mathrm{http}$ ://theconversation.com/robots-are-playing-many-roles-in-the-coronaviruscrisis-and-offering-lessons-for-future-disasters- 135527

Biron, C. L. (2020, July 20). Drones to robots: Pandemic fuels U.S. autonomous delivery. Reuters. https://www.reuters.com/article/us-health-coronavirus-usa-tech-feature-t/drones-to-robotspandemic-fuels-u-s-autonomous-delivery-idUSKCN24L1OI

Blake, R. (2020, April 17). In coronavirus fight, robots report for disinfection duty. Forbes. https://www.forbes.com/sites/richblake1/2020/04/17/ in-covid-19-fight-robots-report-fordisinfection-duty/

Boyle, A. (2020, December 31). FAA issues safety rules that could smooth the way for Amazon drone deliveries. GeekWire. https://www.geekwire.com/2020/faa-issuessafety-rules-smooth-way-amazon-drone-deliveries/

Corkery, M., \& Gelles, D. (2020, April 11). Robots welcome to take over, as pandemic accelerates automation. The New York Times. https://www.nytimes.com/2020/04/10/business/coronavirus-workplaceautomation.html

Demaitre, E. (2020, March). Covid-19 pandemic prompts more robot usage worldwide. The Robot Report. https://www.therobotreport.com/covid-19-pandemic-prompts-morerobot-usage-worldwide/

Eglash, R., Robert, L. P., Bennett, A., Robinson, K. P., Lachney, M., \& Babbitt, W. (2020). Automation for the artisanal economy: Enhancing the economic and environmental sustainability of crafting professions with human-machine collaboration, AI \& Society, 35, 595-609. https://doi.org/10.1007/s00146-019-00915-w

Esterwood, C., \& Robert, L. P. (2020, November). Human robot team design. In Proceedings of the 8th International Conference on Human-Agent Interaction (pp. 251-253).

Federal Aviation Administration. (2020, December 28). Press release - U.S. Department of Transportation issues two much-anticipated drone rules to advance safety and innovation in the United States.

https://www.faa.gov/news/press_releases/news_story.cfm?newsId=25541

Field, H., \& Murphy, M. (2020, April). Covid-19 will herald an automation boom. Protocol.com. https://www.protocol.com/automation-boom-caused-by-coronavirus

Handling, M. M. (2020, July). Other voices: Mobile robots in the time of coronavirus. Modern Materials Handling. https://www.mmh.com/article/other_voices_mobile_robots_in_the_time_of_coronavir $\mathrm{S}$

Hasija, S., \& Gumaledar, A. (2020, April). Are robots overrated? Harvard Business Review. https://hbr.org/2020/04/are-robots-overrated

Kahn, J. (2020, June). Cleaning robot sales are soaring amid the coronavirus pandemic. Fortune. https://fortune.com/2020/06/01/ cleaning-robots-sales-soar-coronavirus-pandemic/

Knight, W. (n.d.). As workers spread out to halt the virus, robots fill the gaps. Wired. https://www.wired.com/story/ workers-spread-halt-virus-robots-fill-gaps/

Marr, B. (2020, May). Demand for these autonomous delivery robots is skyrocketing during this pandemic. Forbes. https://www.forbes.com/sites/bernardmarr/2020/05/29/demand-for-theseautonomous-delivery-robots-is-skyrocketing-during-this-pandemic/ 
Purtill, C. (2020, April). Meet Violet, the robot that can kill the COVID-19 virus. Time. https://time.com/5825860/coronavirus-robot/

PYMNTS. (2019, March). Robots gaining more power in grocery, delivery. PYMNTS.com. https://www.pymnts.com/news/ecommerce/2019/robots-grocery-inventorydelivery/\#: : text=Overall\%2C\%20the $\% 20$ report $\% 20$ found $\% 20 \mathrm{a}, 48 \% 20$ percent $\% 20 \mathrm{y}$ ear\%20over\%20year

Redman, R. (2019, April). Giant Eagle tests shelf-scanning robots. Supermarket News. https://www.supermarketnews.com/retail-financial/ giant-eagle-tests-shelf-scanning-robots

Robert, L. P., Alahmad, R., Kim, S., Esterwood, C., You, S. and Zhang, Q. (2020). A review of personality in human robot interactions. Foundations \& Trends in Information Systems, 4(2), 107-210. http://dx.doi.org/10.1561/2900000018

Seyitoğlu, F., \& Ivanov, S. (2020). Service robots as a tool for physical distancing in tourism. Current Issues in Tourism, 1-4.

Shin, H. (2020, June). Armed with disinfectant and admonishments, South Korean robot fights coronavirus spread. Reuters. https://www.reuters.com/ article/us-health-coronavirussouthkorea-robots-idUSKBN23816M

Shen, Y., Guo, D., Long, F., Mateos, L. A., Ding, H., Xiu, Z., Hellman, R. B., King, A., Chen, S., Zhang, C., \& Tan, H. (2020). Robots under COVID-19 pandemic: A comprehensive survey. IEEE Access. doi:10.1109/ACCESS.2020.3045792

Simon, M. (n.d.). If robots steal so many jobs, why aren't they saving us now? Wired. https://www.wired.com/story/robot-jobs-coronavirus/

Slotkin, J. (2020, April). Meet "Spot": The robot that could help doctors remotely treat COVID-19 patients. NPR. https://www.npr.org/sections/ coronavirus-liveupdates/2020/04/24/844770815/meet-spot-the-robot-that-could-help-doctors-remotely-treatcovid-19-patients

Tangermann, V. (2020, April 10). Demand for autonomous delivery bots is "expanding exponentially." Futurism. https://futurism.com/the-byte/demand-for-autonomous-deliverybots-is-expanding-exponentially

Terra Drone. (2020, February). Terra Drone business partner Antwork helps fight coronavirus in China with medical delivery drones. Terra News. https://www.terradrone.net/global/2020/02/07/terra-drones-business-partner-antwork-helps-fighting-coronavirus-with-drones/

Turmelle, L. (2020, May 08). Don't worry, Stop \& Shop cleans Marty. The Norwalk Hour.

Vincent, J. (2020, May). Spot the robot is reminding parkgoers in Singapore to keep their distance from one another. The Verge. https://www.theverge.com/2020/5/8/21251788/spot-boston-dynamics-robotsingapore-park-social-distancing

Walsh, B. (n.d.). Coronavirus speeds the way for robots in the workplace. Axios. https://www.axios.com/coronavirus-robots-workplace-2654b270-c0cd-4495-82f8ad96fb9663f2.html

World Health Organization. (2020, Dec 27). Coronavirus disease (COVID-19) situation report. https://www.who.int/publications/m/item/weekly-epidemiological-update---29december-2020

Yang, G. Z., Nelson, B. J., Murphy, R. R., Choset, H., Christensen, H., Collins, S. H., Dario, P., Goldberg, K., Ikuta, K., Jacobstein, N., \& Kragic, D. (2020, March). Combating COVID-19 - the role of robotics in managing public health and infectious diseases. Science Robotics, 5 (40). doi:10.1126/scirobotics.abb5589

You, S., Kim, J., Lee, S., Kamat, V., \& Robert, L. P. (2018). Enhancing perceived safety in human-robot collaborative construction using immersive virtual 
environments. Automation in Construction, 96, 161-170.

https://doi.org/10.1016/j.autcon.2018.09.008.

You, S., \& Robert, L. P. (2018a). Emotional attachment, performance, and viability in teams collaborating with embodied physical action (EPA) robots. Journal of the Association for Information Systems, 19(5), 377-407. http://doi.org/10.17705/1jais.00496

You, S., \& Robert Jr., L. P. (2018b, February). Human-robot similarity and willingness to work with a robotic co-worker. In Proceedings of the 2018 ACM/IEEE International Conference on Human-Robot Interaction (pp. 251-260).

Zeldovich, L. (2020, April). Robots fight the coronavirus pandemic. ASME. https://www.asme.org/topics-resources/content/the-robot-will-see-you-now 